\title{
Hepatectomia para o Tratamento de Metástases Colorretais e Não-Colorretais: Análise Comparativa em 30 Casos Operados
}

\author{
Hepatectomy for Metastasis from Colorectal and Non-Colorectal Origin: \\ Comparative Analysis in 30 Resectable Cases
}

\author{
SERGIO RENATO PAIS COSTA ${ }^{1}$; SÉRGIO HENRIQUE HORTA²; ALEXANDRE CRUZ HENRIQUES ${ }^{3}$; \\ JAQUES WAISBERG ${ }^{4}$; MANLIO BASÍLIO SPERANZINI ${ }^{5}$
}

\begin{abstract}
1. Especialista em Cancerologia Cirúrgica - Doutorando e Mestre em Cirurgia pela Universidade Federal de São Paulo - São Paulo - Brasil; ${ }^{2}$ Especialista em Coloproctologia - Médico Assistente do Hospital de Ensino -

Faculdade de Medicina do ABC - Santo André - São Paulo - Brasil; ${ }^{3}$ Chefe do Serviço de Cirurgia Geral do Hospital de Ensino - Faculdade de Medicina do ABC - Santo André - São Paulo - Brasil; ${ }^{4}$ Professor Assistente Doutor da Disciplina de Cirurgia do Aparelho Digestivo - Faculdade de Medicina do ABC - Santo André - São Paulo - Brasil; 5. Professor Titular da Disciplina de Cirurgia do Aparelho Digestivo - Faculdade de Medicina do ABC - Santo André - São Paulo - Brasil.
\end{abstract}

COSTA SRP; HORTA SH; HENRIQUES AC; WAISBERG J; SPERANZINI MB. Hepatectomia para o Tratamento de Metástases Colorretais e Não-Colorretais: Análise Comparativa em 30 Casos Operados. Rev bras Coloproct, 2009;29(2): 216-225.

Resumo: Racional - Hepatectomia é a melhor opção terapêutica curativa para metástases hepáticas de origem colorretal. Mais recentemente, ressecção hepática também tem sido realizada para metástases de etiologia não-colorretal. Objetivo - Comparar os resultados em curto e longo prazo de uma série de hepatectomias para 20 doentes com metástase colorretal com uma série de 10 doentes com metástase não-colorretal realizadas pelo Serviço de Cirurgia Geral (Disciplina de Cirurgia do Aparelho Digestivo) da Faculdade Medicina do ABC (Santo André - Brasil). Métodos - Os dados completos de 30 doentes submetidos à hepatectomia por metástase metacrônica entre o período de Janeiro de 2001 e Setembro de 2007 foram avaliados. Vinte com metástase colorretal (Grupo 1) foram comparados com dez com metástase não-colorretal (Grupo 2). Foi realizada análise multivariada dos fatores prognósticos com o programa Epi-Info para Windows. Resultados - Foram realizadas vinte hepatectomias maiores e dez hepatectomias menores. A morbidade foi similar entre os grupos ( $\mathbf{p}>\mathbf{0 , 0 5})$. A mortalidade cirúrgica foi maior no Grupo 1 do que no Grupo 2 ( $5 \%$ X 0 \%), mas não houve significância estatística (p>0,05). Os índices de sobrevida global em 3 e 5 anos foram comparáveis entre os dois grupos (p>0,05). Conclusão - Nessa amostra, a ressecção hepática para metástase de etiologia nãocolorretal apresenta resultados similares aos da metástase colorretal com sobrevida em cinco anos de $20 \%$. Foram fatores prognósticos adversos: mais que uma metástase e linfonodo positivo.

Descritores: Neoplasias colorretais; Hepatectomia; Metástase neoplásica; Neoplasias hepáticas/cirurgia; Neoplasias hepáticas/secundário; Neoplasias colorretais; Taxa de Sobrevida.

\section{INTRODUÇÃO}

Atualmente, a ressecção cirúrgica de metástases hepáticas de etiologia colorretal é a única modalidade com intenção curativa ou de prolongamento da sobrevida. Em séries mais recentemente publicadas, a sobrevida dos pacientes submetidos à sua ressecção completa (R0) tem variado de $25 \%$ até $55 \%$ em cinco anos (1-3). Em contrapartida, quando indivíduos com disseminação hepática são submetidos a tratamento quimioterápico exclusivo, raramente alcançam mais do que três anos de sobrevida (4).

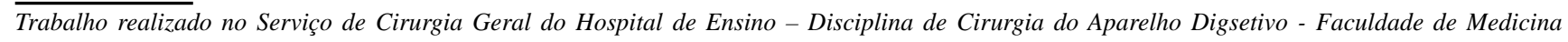
do ABC - Santo André - Brasil. 
Além do melhor conhecimento da biologia tumoral e da diminuição da mortalidade das hepatectomias, a ausência de tratamento com resultado similar contribuiu sobremaneira para ascensão do tratamento operatório. Atualmente, com a evolução de técnicas como ressecções em dois ou mais tempos (com ou sem embolização portal), associação da ressecção com métodos de ablação (notadamente a radiofrequência) e a utilização de quimioterapia neo-adjuvante têm promovido um aumento do entusiasmo na ressecabilidade das metástases. Assim, um aumento da sobrevida mesmo nos enfermos considerados anteriormente como irressecáveis, particularmente naqueles com múltiplas metástases bilaterais tem sido observada (1-8).

Essa recente abordagem, com impacto positivo na sobrevida dos doentes com metástases colorretais (CR) e posteriormente naqueles de origem neuroendócrina (NE), impulsionou sobremaneira a ampliação das indicações das hepatectomias para outros tumores primários metastáticos para o fígado.

Por conseguinte, diversas séries de ressecção de metástases de origem não-colorretal e nãoneuroendócrina (NCRNE) tem sido publicadas. E seus resultados em longo prazo têm sido surpreendentes quando realizadas em casos adequadamente selecionados. Metástases de tumores do trato genito-urinário, de mama e de sarcomas parecem apresentar resultados comparáveis aos de origem CR (9-18). Em nossas meio, pequenas séries de casos de ressecção hepática de metástases de sarcomas, mama e também de outros primários tem sido também descritas (19-21). Paralelamente, poucos estudos em nosso país foram realizados com o objetivo de avaliar possíveis fatores prognósticos em longo prazo mesmo para metástases de origem CR $(22,23)$. Particularmente, não foi encontrado estudo comparativo em nosso meio em relação aos resultados em curto e longo prazo das hepatectomias para tratamento de metástases de etiologia CR e NCRNE, e avaliação de possíveis fatores prognósticos.

Assim, o objetivo do presente estudo foi comparar os resultados do tratamento cirúrgico radical dos indivíduos portadores de metástases hepáticas CR com aqueles com metástases NCRNE e identificar possíveis fatores prognósticos.

\section{MÉTODOS}

Durante o período de Janeiro de 2001 a Setembro de 2007 foram diagnosticados 44 doentes com metástases hepáticas metacrônicas de etiologia CR, NE e NCRNE no Hospital de Ensino da Faculdade de Medicina do ABC, Santo André - São Paulo. Todos doentes foram avaliados e operados por uma mesma equipe cirúrgica do Serviço de Cirurgia Geral do Hospital de Ensino - Disciplina de Cirurgia do Aparelho Digestivo da Faculdade de Medicina do ABC, Santo André - São Paulo.

Nove doentes foram excluídos do tratamento cirúrgico por falta de condições clínicas para ressecção $(n=2)$, doença hepática extensa bilateral $(n=2)$, doença extra-hepática irressecável $(n=2)$, doença extensa sincrônica $(n=3)$. No total, trinta e cinco foram submetidos à exploração cirúrgica. Contudo, em três foi documentada carcinomatose ao exame de congelação intra-operatória, e foram excluídos dessa amostra. Dois outros doentes, também foram excluídos da casuística por serem portadores de metástases de etiologia NE e por terem sido submetidos a cirurgias com intuito paliativo (R2).

Dessa maneira, trinta doentes com metástases metacrônicas (mais que seis meses do tratamento do tumor primário) foram estudados e subdivididos em dois grupos: Grupo 1 de 20 indivíduos portadores de metástases CR e Grupo 2 - 10 indivíduos portadores de metástases NCRNE. As características clínicoepidemiológicas estão demonstradas na Tabela 1.

A maioria dos pacientes era assintomática em relação às metástases hepáticas, exceto no Grupo 1 onde dois doentes apresentavam algum sintoma. Os sintomas apresentados foram: tumoração abdominal $(n=1)$ e dor $(n=1)$.

O número de lesões variou de uma a quatro. No grupo 1, dois doentes apresentavam metástases bilaterais, enquanto que no grupo 2 nenhum doente apresentava bilateralidade. Em todos pacientes do Grupo 1, o diagnóstico histológico foi de adenocarcinoma. Em contrapartida, no Grupo 2 foram encontrados: adenocarcinoma $(n=7)$, carcinoma germinativo $(n=1)$, melanoma $(n=1)$ e sarcoma $(n=1)$. A distribuição do número, tamanho e localização das metástases em cada grupo também está demonstrada na Tabela 1.

$\mathrm{O}$ intervalo livre de doença entre o tratamento da lesão primária e o aparecimento das metástases variou de 12 a 34 meses com mediana de 18 meses para as metástases CR, e de 12 a 30 meses com mediana de 20 meses para as metástases NCRNE (Tabela 1). Nas metástases CR, o tumor primário foi de cólon em 12 doentes (cólon direito em 5, cólon esquerdo em 
Tabela 1 - Características clínico-epidemiológicas dos doentes submetidos a tratamento cirúrgico.

\begin{tabular}{|c|c|c|c|}
\hline & Grupo 1 CR N (\%) & Grupo 2 NCRNE N (\%) & $p$ \\
\hline Número de doentes & 20 & 10 & ns \\
\hline \multicolumn{4}{|l|}{ Sexo } \\
\hline Masculino & $12 \quad(60)$ & $5 \quad(50)$ & ns \\
\hline Feminino & $8 \quad(40)$ & $5 \quad(50)$ & ns \\
\hline Idade & 58 & 53 & ns \\
\hline (mediana - anos) & $\left(\begin{array}{ll}35- & 78\end{array}\right)$ & $(29-68)$ & \\
\hline \multicolumn{4}{|l|}{ Raça } \\
\hline Caucasiana & $12 \quad(60)$ & $6 \quad(60)$ & ns \\
\hline Não-Caucasiana & $8 \quad(40)$ & $4 \quad(40)$ & ns \\
\hline \multicolumn{4}{|l|}{ Classificação ASA } \\
\hline ASA I & $15 \quad(75)$ & $8 \quad(80)$ & ns \\
\hline ASA II-III & $5 \quad(25)$ & $2(20)$ & ns \\
\hline \multicolumn{4}{|l|}{ Número de metástases } \\
\hline 1 & $8 \quad(40)$ & $7 \quad(70)$ & $<0,01$ \\
\hline$>1$ & $12 \quad(60)$ & $3 \quad(30)$ & $<0,01$ \\
\hline Tamanho das metástases & 4,5 & 5 & ns \\
\hline$($ mediana $-\mathrm{cm})$ & $(1-12)$ & $(4-10)$ & \\
\hline Intervalo de aparecimento das metástases & 17 & 20 & ns \\
\hline (mediana - meses) & $(7-30)$ & $(12-30)$ & \\
\hline Albumina pré-operatória & 3,7 & 3,6 & ns \\
\hline$($ mediana $-\mathrm{g} / \mathrm{dl})$ & $(2,8-5,5)$ & $(2,9-5,4)$ & \\
\hline Índice de massa corpórea & 24,3 & 25,1 & ns \\
\hline Quimioterapia & $(18,5-37)$ & $(19,5-36,8)$ & \\
\hline CEA & $15 \quad(75)$ & $7 \quad(70)$ & ns \\
\hline (mediana-ng/dl) & $56,3 \quad(0,5-348)$ & $3,5 \quad(0,2-89,3)$ & $<0,001$ \\
\hline
\end{tabular}

3 e sigmóide em 4) e de reto em 8 . Nas metástases NCRNE, os sítios primários foram: gástrico $(\mathrm{N}=1)$, rim $(\mathrm{N}=1)$, adrenal $(\mathrm{N}=1)$, mama $(\mathrm{N}=2)$, testículo $(\mathrm{N}=1)$, ovário $(\mathrm{N}=2)$, melanoma acral $(\mathrm{N}=1)$ e sarcoma de retroperitônio $(\mathrm{N}=1)$.

Todos pacientes da presente casuística foram submetidos à estadiamento completo pré-operatório com tomografia de abdome, pelve e tórax.

Nos últimos vinte e cinco pacientes foi também realizada ressonância nuclear magnética de abdome pré-operatória. A ultra-sonografia intra-operatória do fígado foi possível de ser realizada em 15 pacientes. Também foram dosados os níveis séricos de CEA, CA 19-9, CA 125, CA 15-3, alfa-fetoproteína em todos os casos.

Após o tratamento cirúrgico, todos pacientes foram acompanhados pelos Serviços de Oncologia Clínica e Cirurgia Geral, por meio de um fichário padroni- zado e todos os dados foram colhidos de forma prospectiva. Os pacientes foram acompanhados segundo a seguinte padronização: intervalos de três meses (até dois anos), de seis meses (entre dois e cinco anos), e finalmente a cada doze meses (após cinco anos). Em cada retorno, além do exame físico completo foram dosados marcadores conforme a origem do primário, ultra-sonografia total de abdome, tomografia computa-dorizada de abdome-pelve e raios-X simples de tórax. Recorrência tumoral foi considerada somente em casos de documentação radiológica inequívoca, e se possível com confirmação histológica.

Para avaliação estatística foi utilizado o Programa EPI-INFO para Windows. Foi realizada análise uni e multivariada dos diferentes fatores prognósticos. Foi considerado como estatisticamente significante um $\mathrm{p}<0,05$. O tempo de sobrevida foi calculado da data da ressecção da metástase até o último retorno 
ambulatorial ou morte. A sobrevida global, sobrevida livre de doença e controle local foram calculados usando método de Kaplan-Meier.

\section{RESULTADOS}

Não houve diferença estatisticamente significativa entre os grupos 1 e 2; em relação ao tipo de hepatectomia (maior ou menor), tempo cirúrgico, sangramento estimado, necessidade de transfusão e tempo de isquemia hepática (Tabela 2). Quatorze doentes apresentaram no mínimo uma complicação e a morbidade global dessa série foi de $46 \%$. Não houve diferença estatisticamente significativa entre os grupos 1e 2, em relação a essa variável. Houve seis complicações maiores (complicações graves tanto clínicas com risco de morte quanto cirúrgicas com necessidade de reoperação) e oito menores. As complicações maiores apresentadas foram: abscesso intracavitário $(n=2)$, fístula biliar $(n=2)$, evisceração $(n=1)$ e insuficiência hepática $(n=1)$. Cinco doentes foram submetidos à reintervenção cirúrgica (16\%). Não houve diferença estatisticamente significativa entre os grupos 1 e 2 , em relação ao número de reoperações. Dentre as complicações menores foram observadas: pneumonia $(n=4)$, infecção urinária $(n=1)$, derrame pleural $(n=1)$, infec- ção de parede $(n=1)$, seroma $(n=1)$. O tempo de internação foi similar entre os grupos ( $p>0,05)$. No grupo 1 , um paciente submetido à hepatectomia direita ampliada para o segmento IV com ressecção parcial do diafragma e interposição de pericárdio bovino por uma metástase de $12 \mathrm{~cm}$ faleceu por insuficiência hepática pós-operatória totalizando uma mortalidade de 5\%, enquanto no Grupo 2 não houve mortalidade. Contudo não se observou diferença estatisticamente significativa em relação a essa variável (Tabela 3 ).

No Grupo 1, dezoito doentes apresentaram margens microscópicas livres (R0), enquanto dois apresentaram margens microscopicamente comprometidas (R1). No Grupo 2, todos pacientes doentes apresentaram margens livres (R0). Não houve diferença estatisticamente significativa em relação a essa variável $(\mathrm{p}$ $>0,05$ ). Os últimos 25 doentes da presente casuística foram submetidos à linfadenectomia hilar sistemática sendo 15 no Grupo 1 e 10 no Grupo 2. A mediana de linfonodos dissecados foi de cinco (variando de um a nove). Oito doentes apresentaram pelo menos um linfonodo comprometido (32\%). O grupo 2 apresentou proporcionalmente mais doentes com comprometimento linfonodal (40\% X 26\%). No entanto, essa diferença estatisticamente significativa ( $p>0,05)$. Essas variáveis podem ser avaliadas na Tabela 3 .

Tabela 2 - Características das cirurgias realizadas.

\begin{tabular}{|c|c|c|c|}
\hline & Grupo 1 CR N (\%) & Grupo 2 NCRNE N (\%) & $p$ \\
\hline Número de doentes & 20 & 10 & \\
\hline \multicolumn{4}{|l|}{ Hepatectomia } \\
\hline Maior & $13 \quad(65)$ & $7 \quad(70)$ & $\mathrm{ns}$ \\
\hline Menor & $7 \quad(35)$ & $3 \quad(30)$ & $\mathrm{ns}$ \\
\hline $\begin{array}{l}\text { Tempo Cirúrgico } \\
\text { (mediana - minutos) }\end{array}$ & $\begin{array}{l}250 \\
(120-478)\end{array}$ & $\begin{array}{l}240 \\
(180-425)\end{array}$ & $\mathrm{ns}$ \\
\hline \multicolumn{4}{|l|}{ Transfusão } \\
\hline Não transfundidos & $12 \quad(60)$ & $5 \quad(50)$ & ns \\
\hline$<1000 \mathrm{ml}$ & $4 \quad(20)$ & $3 \quad(30)$ & $\mathrm{ns}$ \\
\hline$>1000 \mathrm{ml}$ & $4 \quad(20)$ & $2(20)$ & $\mathrm{ns}$ \\
\hline $\begin{array}{l}\text { Sangramento Estimado intra-operatório } \\
\text { (mediana }-\mathrm{ml})\end{array}$ & $\begin{array}{l}1000 \\
(0-4500)\end{array}$ & $\begin{array}{l}800 \\
(200-3000)\end{array}$ & $\begin{array}{l}\mathrm{ns} \\
\mathrm{ns}\end{array}$ \\
\hline $\begin{array}{l}\text { Manobra de Pringle } \\
\text { (número de doentes) }\end{array}$ & & & \\
\hline Sem Clampeamento & $5 \quad(25)$ & $2(20)$ & ns \\
\hline$<30$ minutos & $10 \quad(50)$ & $5 \quad(50)$ & $\mathrm{ns}$ \\
\hline$>30$ minutos & $5 \quad(25)$ & $3 \quad(30)$ & $\mathrm{ns}$ \\
\hline
\end{tabular}


Tabela 3 - Resultados precoces.

\begin{tabular}{|c|c|c|c|}
\hline & Grupo 1 CR N (\%) & Grupo 2 NCRNE N (\%) & $p$ \\
\hline Número de doentes & 20 & 10 & \\
\hline \multicolumn{4}{|l|}{ Complicações } \\
\hline Maiores & $4 \quad(20)$ & $2(20)$ & ns \\
\hline Menores & $5 \quad(25)$ & $3 \quad(30)$ & ns \\
\hline Reoperação & $3 \quad(15)$ & $2(20)$ & ns \\
\hline Tempo internação & 250 & 240 & \\
\hline (mediana - minutos) & $(120-480)$ & $(180-425)$ & $\mathrm{ns}$ \\
\hline Mortalidade & $1 \quad(5)$ & $\begin{array}{ll}0 & (0)\end{array}$ & ns \\
\hline \multicolumn{4}{|l|}{ Tumor (Grau) } \\
\hline Alto & $10 \quad(33)$ & $3 \quad(30)$ & ns \\
\hline Moderado-baixo & $20 \quad(67)$ & $7 \quad(70)$ & ns \\
\hline \multicolumn{4}{|l|}{ Margens } \\
\hline R0 & $18 \quad(90)$ & $10(100)$ & $\mathrm{ns}$ \\
\hline R1 & $2 \quad(10)$ & $0 \quad(0)$ & $\mathrm{ns}$ \\
\hline \multicolumn{4}{|l|}{ Linfonodo Hilar } \\
\hline Positivo & $4 \quad(26)$ & $4 \quad(40)$ & ns \\
\hline Negativo & $11 \quad(74)$ & $6 \quad(60)$ & $\mathrm{ns}$ \\
\hline
\end{tabular}

O período de seguimento dos pacientes variou de 13 a 60 meses, com mediana de 38 meses. Quinze doentes apresentaram recidivas. Oito doentes apresentaram recidiva exclusivamente hepática, enquanto sete doentes apresentaram recidiva múltipla. O Grupo 1, apresentou proporcionalmente mais recidivas hepáticas $(p<0,05)$. Em contrapartida, o Grupo 2 apresentou mais recidivas múltiplas $(\mathrm{p}<0,05)$. No Grupo 2, dois doentes foram submetidos a hepatectomia de resgate. $\mathrm{O}$ intervalo entre a cirurgia hepática e a recidiva variou de 10 a 33 meses. A sobrevida global em três anos foi de $50 \%$, enquanto em cinco anos foi de $20 \%$. Não houve diferença estatisticamente significativa entre os Grupos 1 e 2 em relação a sobrevida aos três e cinco anos de sobrevida. Contudo, no Grupo 2, a sobrevida livre de doença foi estatisticamente menor (Tabela 4).

Na Tabela 5 estão demonstradas as características epidemiológicas dos 14 doentes com sobrevida livre de doença maior do que 36 meses. Ao se realizar

Tabela 4 - Resultados em longo prazo.

\begin{tabular}{|c|c|c|c|}
\hline & Grupo 1 CR N (\%) & Grupo 2 NCRNE N (\%) & $p$ \\
\hline Número de doentes & 20 & 10 & \\
\hline \multicolumn{4}{|l|}{ Sobrevida global } \\
\hline Em 3 anos & $11 \quad(57)$ & $5 \quad(50)$ & ns \\
\hline Em 5 anos & $4 \quad(21)$ & $2(20)$ & ns \\
\hline \multicolumn{4}{|l|}{ Sobrevida Livre de Doença } \\
\hline Em 3 anos & $10 \quad(52)$ & $3(30)$ & $<0,05$ \\
\hline Em 5 anos & $4 \quad(21)$ & $0 \quad(0)$ & $<0,01$ \\
\hline Mediana de Sobrevida (meses) & 38 & 34 & ns \\
\hline Número de doentes com recidiva $(n=15)$ & $10 \quad(50)$ & $5 \quad(50)$ & ns \\
\hline Recidiva Hepática Exclusiva $(\mathrm{n}=8)$ & $7 \quad(40)$ & $1 \quad(10)$ & $<0,03$ \\
\hline
\end{tabular}


a análise univariada, as seguintes variáveis independentes tiveram impacto negativo na sobrevida em longo prazo dos doentes: idade superior 70 anos, CEA maior que $200 \mathrm{ng} / \mathrm{dl}$, transfusão intra-operatória, mais que uma lesão hepática, tamanho da lesão maior que 5 $\mathrm{cm}$ e linfonodo positivo no tumor primário ou no hilo hepático. Contudo, a análise multivariada somente número de lesões (mais que uma) e linfonodo positivo (primário ou no hilo hepático) foram considerados fatores de mau prognóstico para sobrevida em longo prazo $(\mathrm{p}<0,01)$.

\section{DISCUSSÃO}

Considerando-se que o fígado é um órgão frequentemente acometido por metástases, os mecanismos para o seu acometimento ainda é motivo de estudo e tende a diferir segundo o sítio primário do tumor. A via mais comum de disseminação em doentes com tumores oriundos do trato gastrointestinal (como o câncer colorretal e tumores neuroendócrinos do trato digestório) são a drenagem venosa pela veia porta e a via retrógrada pelos canais linfáticos. O racional da ressecção das metástases hepáticas seria que teoricamente a doença estaria confinada a um sítio loco-regional (cavidade abdominal). Assim sendo, o tratamento radical e apropriado tanto do tumor primário quanto das metástases pode teoricamente oferecer chances de controle ou mesmo cura da neoplasia. Do ponto de vista prático, este raciocínio tem sido confirmado, ami- úde em metástases CR, onde $37 \%$ dos doentes sobrevivem cinco anos e pelo menos $22 \%$ sobrevivem dez anos (24).

Com o melhor conhecimento da biologia tumoral, da técnica cirúrgica das hepatectomias, das condições de suporte intra e pós-operatórias (principalmente a partir das décadas de 80 e 90) houve um crescente entusiasmo no tratamento das metástases hepáticas. Fortner et al. (25), já na década de 80 sinalizava a hepatectomia como tratamento curativo das metástases hepáticas colorretais. A partir deste autor que estudou uma casuística de 247 indivíduos operados foi possível de se conhecer os primórdios do entendimento da biologia tumoral das metástases CR e de seu tratamento cirúrgico.

Desde então, diversas séries foram importantes para o melhor conhecimento deste tipo de procedimento, o que culminou com uma rápida expansão das indicações desta terapêutica, principalmente em virtude de seus bons resultados quando comparados ao tratamento sistêmico exclusivo (26-31). Paralelamente, com o desenvolvimento técnico, de suporte pré e pósoperatório a mortalidade das ressecções hepáticas foram diminuindo (8).

Por conseguinte, atualmente, a ressecção curativa de metástases hepática de origem $\mathrm{CR}$ tem sido considerada padrão ouro, e mesmo na presença de fatores prognósticos negativos a sobrevida em alguns enfermos pode ser eventualmente prolongada. Séries

Tabela 5 - Características dos sobreviventes livres de doença (mais 36 meses).

\begin{tabular}{|c|c|c|c|c|c|c|c|c|c|c|c|c|c|c|}
\hline$\overline{\text { Caso }}$ & 1 & 2 & 3 & 4 & 5 & 6 & 7 & 8 & 9 & 10 & 11 & 12 & 13 & 14 \\
\hline Primário & $\mathrm{C}$ & $\mathrm{R}$ & $\mathrm{C}$ & $\mathrm{C}$ & $\mathrm{C}$ & $\mathrm{R}$ & $\mathrm{C}$ & $\mathrm{R}$ & $\mathrm{R}$ & $\mathrm{C}$ & $\mathrm{M}$ & M & $\mathrm{T}$ & A \\
\hline $\begin{array}{l}\text { Maior Diâmetro } \\
\text { de lesão }(\mathrm{cm})\end{array}$ & 3 & 3,5 & 4 & 2 & 6 & 3 & 2,5 & 5 & 4 & 5 & 4 & 10 & 8 & 8 \\
\hline $\begin{array}{l}\text { Número de lesões } \\
\text { linfonodo }\end{array}$ & 1 & 1 & 1 & 1 & 1 & 1 & 1 & 2 & 2 & 1 & 2 & 1 & 1 & 1 \\
\hline (tu primário) & - & - & - & - & - & - & + & - & + & - & - & - & - & - \\
\hline Transfusão & - & - & - & + & + & - & - & + & - & - & + & + & - & + \\
\hline Marcador tu > valor $\mathrm{nl}$ & - & - & + & + & + & + & - & + & - & - & + & + & - & + \\
\hline $\begin{array}{l}\text { Grau de diferenciação } \\
\text { linfonodo }\end{array}$ & B & B & $\mathrm{M}$ & $\mathrm{M}$ & B & $\mathrm{M}$ & B & $\mathrm{M}$ & B & B & B & B & $\mathrm{M}$ & B \\
\hline (hilar) & - & - & - & - & - & - & - & - & - & - & - & - & - & - \\
\hline Sobrevida (m) & 40 & 38 & 37 & 50 & 55 & 41 & 44 & 39 & 56 & 60 & 48 & 40 & 50 & 43 \\
\hline
\end{tabular}

C-Cólon, R-Reto, M-Mama, A-Adrenal, T-Testículo, + positivo, - negativo, cm-centimetros, tu-tumor, nl-normal, B-baixo, M-Moderado. 
históricas importantes foram reportadas o que culminou com o melhor conhecimento dos fatores prognósticos levando consequentemente a uma melhor seleção do doente com metástase CR candidato ao tratamento cirúrgico. Nordingler et al (29), em estudo multicêntrico na França em 85 centros de excelência com casuística total de 1568 doentes operados, observaram uma mortalidade operatória de $2,3 \%$ sendo estatisticamente maior nos idosos (idade maior 60 anos) e nas hepatectomias maiores ou nas metástases maiores que $5 \mathrm{~cm}$. A morbidade global foi de $23 \%$, e foram mais comumente encontradas em hepatectomias maiores. À análise multivariada, dentre os fatores prognósticos adversos independentes, foram observados: idade maior que 60 anos, comprometimento da serosa, linfonodo positivo, intervalo livre de doença entre o tratamento do primário e aparecimento de metástase menor que dois anos, tamanho da maior lesão (maior que cinco $\mathrm{cm}$ ), número de lesões (maior que quatro) e margem cirúrgica menor que um $\mathrm{cm}$. A sobrevida global foi de $25 \%$.

Em outra grande série do Memorial SloanKettering Cancer Center, Fong et al (24), ao avaliarem 1001 indivíduos operados em um único centro observaram mortalidade e morbidade operatória de $3,8 \%$ e $31 \%$ respectivamente, e idealizaram um escore clínico preditivo de sobrevida. À análise multivariada sete fatores negativos para sobrevida em longo prazo foram encontrados: margens positivas, doença extra-hepática, status linfonodal do tumor primário, intervalo livre doença entre o tumor primário e aparecimento da metástase (menor que um ano), número de metástases (maior que um), tamanho da maior lesão (maior que $5 \mathrm{~cm}$ ) e antígeno carcinoembrionário maior que $200 \mathrm{ng} / \mathrm{ml}$. Quando cinco destes critérios eram positivos apenas $14 \%$ dos doentes sobreviveram mais que cinco anos (mediana de sobrevida de 22 meses), e ao contrário quando todos eram negativos $60 \%$ dos doentes sobreviveram pelo menos cinco anos (mediana de sobrevida de 74 meses).

Em nosso meio, Oliveira et al (23) ao analisarem 41 doentes submetidos a ressecção curativa (todos com menos que quatro metástases) com mortalidade pós-operatória nula, observaram que apenas a localização no reto foi fator prognóstico reservado. A média de sobrevida foi de 38,4 meses. Chedid et al (22), ao analisarem 28 doentes operados com mortalidade de $3,6 \%$ e morbidade de $39,3 \%$, observaram sobrevida de $35 \%$ em cinco anos. A análise multivariada encontrou como fatores prognósticos adversos independentes, apenas intervalo livre de doença menor que um ano e a presença de doença extra-hepática.

No presente estudo a mortalidade (3\%) e morbidade $(46 \%)$ foram similares as encontradas na literatura, e não houve diferença estatisticamente significante entre essas variáveis em relação aos grupos (1-CR e 2-NCRNE), fato este também observado previamente por Reddy et al (32). O único caso de óbito foi em doente submetido à hepatectomia direita ampliada com ressecção do diafragma, e como já descrito por Nordingler et al (29) é fato conhecido que uma pluri-segmentectomias tem mortalidade estatisticamente maior quando comparada as ressecções menores (menos que 3 segmentos).

No presente estudo, os candidatos a ressecção foram rigorosamente selecionados levando-se em conta os fatores prognósticos previamente descritos tanto para metástases CR quanto NCRNE. Dentre os mais importantes a nosso ver e que foram seguidos a risca foram: intervalo livre de doença e número de lesões. Somente foram operados doentes com lesões metacrônicas (intervalo livre de doença maior que seis meses) e de preferência doentes com lesão única e no máximo quatro, isto baseado em diversos estudos que demonstraram baixa sobrevida para indivíduos com metástases sincrônicas $(2,6,8,10,11,13,14,24,29)$. Fato que foi confirmado nesse estudo, onde o número de lesões (maior que uma) foi uma variável independente no prognóstico. Assim como Nordingler et al (29) e Fong et al (24), a presença de linfonodo comprometido no tumor primário $\mathrm{CR}$ também tem sido implicada no mau prognóstico como pode ser observada na presente casuística.

Quando analisados também os NCRNE podese observar que linfonodos comprometidos também tiveram impacto negativo na sobrevida. Dos quatorze doentes que sobreviveram mais que 36 meses, apenas dois ambos do Grupo 1 (CR) apresentavam linfonodos comprometidos no tumor primário, e nenhum do Grupo 2 (NCRNE). Paralelamente, presença de linfonodo hilar comprometido também tem sido motivo de estudos. Dúvidas pairam em relação ao benefício da hepatectomia frente ao comprometimento de linfonodo hilar podendo inclusive ser causa de contra-indicação a cirurgia radical $(33,34)$. Segundo revisão sistemática 
realizada por Rodgers \& McCall (35), a chance de sobrevida em cinco anos para doentes com linfonodo positivo hilar, seja em uma dissecção sistemática ou não durante a hepatectomia para metástases CR, é muito pequena. Para metástases NCRNE, embora não tenhamos encontrado muitos estudos com esse objetivo, baseado na presente amostra mesmo que pequena pudemos observar que metástase linfonodal hilar no grupo 2 (NCRNE), também foi considerada de prognóstico reservado a análise multivariada denotando uma doença sistêmica "a priori". Especificamente para metástases de mama, Sakamoto et al (36) em casuística de 34 doentes ressecados não observaram nenhum sobrevivente com mais de cinco anos com linfonodo hilar positivo. Este autor descreveu a presença de linfonodo hilar positivo como variável adversa no prognóstico final. Dados similares foram encontrados na presente casuística. Dos 25 doentes submetidos à dissecção hilar sistemática, pode-se observar que de todos os que apresentaram gânglio hilar positivo $(n=8)$, nenhum atingiu uma sobrevida maior que 20 meses independentemente do grupo.

Embora a casuística tenha sido pequena, não pode ser observada diferença estatisticamente significante na sobrevida entre doentes com metástases CR e NCRNE, ou seja, elas foram muito similares (em torno de $50 \%$ e $20 \%$ em três e cinco anos respectivamente). Isto pode ser devido à intensa seleção dos casos operados (lesões metacrônicas, únicas e tumores do trato genito-urinário e mama predominantemente). Do ponto de vista prático, a ressecção de metástases NCRNE tem sido fortemente recomendada em casos selecionados na última década. Grandes casuísticas em instituições renomadas e estudos de fatores prognósticos têm sido sistematicamente publicados. Os índices de sobrevida nas últimas séries tem se aproximado dos índices publicados nas de metástases CR e tem variado de 24 até $37 \%$ em cinco anos $(22,37,38)$. A sobrevida global de 20 $\%$ em cinco anos da presente série, tanto no Grupo 1 (CR) quanto no Grupo 2 (NCRNE) foi similar a de diversas outras séries previamente descritas $(1,2,14$, 37, 39).

Em relação às metástases NCRNE, a maioria dos estudos tem observado que a sobrevida pode variar conforme o sub-grupo analisado sendo maior nos tumores de mama, genito-urinários e sarcomas que em relação aos demais grupos (tumores do trato gastrointestinal, melanoma não-ocular). Também, o número de metástases, radicalidade da cirurgia (R0) bem como o intervalo de doença entre o primário e a metástase (maior que uma ano, dois ou até mesmo três) tem impacto na sobrevida em longo prazo (12-14, 22, $37,38)$.

$\mathrm{Na}$ presente série, entre os sobreviventes em longo prazo (mais de três anos), todos os primários apresentavam mais que um ano entre seu aparecimento e o surgimento da metástase que em geral foi única (somente uma doente apresentava mais que uma) e também apresentaram distribuição condizente com o observado nos estudos previamente (predomínio de tumores de mama e trato genito-urinário). Assim como observado por Reddy et al (32) ao comparar 245 casos de metástases CR com 82 de metástases NCRNE durante um mesmo período, no presente estudo a sobrevida global foi similar entre os grupos. No entanto, também como descrito por este autor, a sobrevida livre de doença foi estatisticamente menor no grupo NCRNE. No grupo 2 (NCRNE) nenhum dos sobreviventes em cinco anos ( 2 doentes) se apresentava sem neoplasia ao término do estudo. Ao contrário do grupo 1 (CR), onde todos os quatro doentes se apresentavam livres de doença há mais de cinco anos ao término do estudo. Talvez, isto corrobore com um caráter mais sistêmico das metástases NCRNE, levando a crer que esses casos têm que ser ainda mais intensamente selecionados. Fato esse não tão frequentemente observado nas metástases $\mathrm{CR}$ onde um envolvimento mais loco - regional pode ser observado, haja visto que as recidivas mais frequentemente observadas nesse sub-grupo no presente estudo foram mais hepáticas e não sistêmicas.

\section{CONCLUSÕES}

Na presente amostra, a sobrevida dos doentes submetidos à ressecção de metástases não-colorretal e não-neuroendócrina foi semelhante à de doentes com metástases colorretais ( $20 \%$ em cinco anos). No entanto, a sobrevida livre de doença foi estatisticamente menor no grupo NCRNE. Foram fatores prognósticos adversos para a sobrevida pela analise multivariada: comprometimento linfonodal (tumor primário ou linfonodo hilo hepático) e número de lesões (mais que uma). 
ABSTRACT: Background - Hepatectomy has presented the best curative therapeutic choice for hepatic metastatic colorectal cancer. More recently, hepatic resection has been performed for hepatic metastases from non-colorectal origin too. Aim -To compare both early and long-term surgical outcomes on 20 patients' series of colorectal with 10 patients' series of non-colorectal hepatic metastases realized by General Surgery Service (Discipline of Tract Digestive Surgery) of ABC Medical School (Santo André - Brazil). Methods - Complete follow-up data were available on 30 patients who underwent hepatectomy for metastatic methacronic cancer between January 2001 and September 2007. Twenty patients presented colorectal liver metastases (Group 1) were compared with ten patients presented non-colorectal metastases (Group 2). Results - There were twenty major hepatic resections and ten minor hepatic resections. Overall morbidity rates were similar between Groups 1 and $2(p=n s)$. Overall mortality in Group 1 was higher than Group 2 (5\% X 0 \%), nevertheless there was no statistical significance (p=ns). Both 3 and 5-year overall survival rates were comparable between groups $(p=n s)$. Both number of lesions and nodal disease were considered dismal prognostic factors. Conclusion - In this sample, hepatic resection for liver metastasis from non-colorectal and nonneuroendocrine origin presents similar results to colorectal metastasis. Multiple metastases and positive node were adverse prognostic factors.

Key words: Colorrectal neoplasm; Hepatectomy; Neoplasm metastasis; Liver neoplasm/surgery; Liver neoplasm/secondary; Survival rate.

\section{REFERÊNCIAS}

1. Adam R, Laurent A, Azoulay D, Castaign D, Bismuth H. Two-Stage hepatectomy: A Planned strategy to Treat Irresectable Liver Tumors. Ann Surg 2000; 232(6): 777-785.

2. Adam R. Tratamento das Metástases Hepáticas do Câncer Colorretal. In: Correia MM, Mello ELR, Santos CER. Eds. Cirurgia do Câncer Hepatobiliar. $1^{\circ}$ Edição, Rio de Janeiro. Revinter; 2003: p. 139-146.

3. Azoulay D, Castaign, Smail A, Adam R, Cailliez V, Laurent A, Lemoine A, Bismuth H. Resection of Nonresectable Liver Metastases From Colorectal Cancer After Percutaneous Portal Vein Embolization. Ann Surg 2000; 231(4): 480-486.

4. Chiche L. Prise en charge chirurgicale des métastases hépatiques des cancers colorectaux. J Chir 2003; 140 (2): 77-89.

5. Jaeck D, Bachellier P, Nakano H, Oussoultzoglou E, Weber JC, Greget M. One or Two-stage hepatectomy combined with portal embolization for initially nonresectable colorectal liver metastases. The Am J Surg 2003; 185: 221-229.

6. Choi EA, Rodgers SE, Ahmad SA, Abdalla EK. Hepatobiliary Cancers. In: Feig BW, Berger DH, Fuhrman GM Eds. The M. D. Anderson Surgical Oncology Handbook. 4 Th Edition, Philadelphia: Lippincott Williams \& Wilkins; 2006. p. 23847.

7. Minagawa M, Makuuchi M, Torzilli G, Takayama T, Kawasaki S, Kosuge T, Yamamoto J, Imamura H. Extension of the Frontiers of Surgical Indications in the Treatment of Liver Metastases from Colorectal Cancer. Ann Surg 2000; 231(4): 487-499.

8. Scheele J, Altendorf-Hofmann A. Surgical treatment of liver metastases. In: Blumgart LH, Fong Y. Ed. Surgery of the Liver and Biliary Tract. 3th edition. Edinburgh WB Saunders 2000: 1475-1502.

9. Schwartz SI. Hepatic resection for noncolorectal nonneuroendocrine metastases. World J Surg 1995; 19: 72-5.
10. Harrisson LE, Brenan MF, Newman E. Hepatic resection for noncolorectal, nonneuroendocrine metastases: a fifteen-year experience with ninety-six patients. Surgery 1997; 121: 625632.

11. Elias D, Cavalcanti de Albuquerque A, Eggenspieler P. Resection of liver metástases from a noncolorectal primary: indications and results based on 147 monocentric patients. J Am Coll Surg; 1998: 187: 493-8.

12. Yamada H, Katoh H, Kondo S. Hepatectomy for metastases from non-colorectal and non-neuroendocrine tumor. Anticancer Res 2001; 21: 4159-62.

13. Laurent C, Rullier E, Feyler A. resection of noncolorectal and nonneuroendocrine liver metastases: late metastases are the only chance of cure. World J Surg 2001: 25: 1532-1536.

14. Weitz J, Blumgart LH, Fong Y, Jarnargin WR, DAngelica M, Harrisson LE, Dematteo RP. Partial Hepatectomy for Metastases From Noncolorectal, Nonneuroendocrine Carcinoma. Ann Surg 2005; 241 (2): 269-276.

15. Karavias DD, Tepetes K, Karatzas T, Felekouras E, Androulakis. Liver resection for non-colorectal nonneuroendocrine hepatic neoplasms. Eur J Surg Oncol, 2002; 28: 135-139.

16. Lang H, Nussbaun KT, Kaudel P, Fruhauf N, Flemming P, Raab R. Hepatic metastases from leiomyosarcoma: A singlecenter experience with 34 liver resections during a15-year period. Ann Surg 2000: 231; 500-5.

17. Dematteo RP, Shah A, Fong Y, Jarnargin WR, Blumgart LH, Brennan MF. Results of hepatic resection for sarcoma metastatic to the liver. Ann Surg 2001; 234: 540-8.

18. Pocard M, Pouillart P, Asselain B, Salmon R. Hepatic resection in metastatic breast cancer: results and prognostic factors. Eur J Surg Oncol 2000; 26: 155-9.

19. Silva RG, Paiva RKS, Santos CER, Dias JA, Bravo Neto GP. Ressecção hepática de sarcomas de partes moles. Rev Col Bras Cir 2006; 33 (6): 380-6. 
20. Kalil NA, Severa C. Hepatectomia para metástases hepáticas de câncer de mama. Rev Col Bras Cir 2005; 32 (5): 285-6.

21. Costa SRP, Horta SHC, Miotto MJ, Costas MC, Henriques AC, Speranzini MB. Ressecção hepática para o tratamento de metástases de origem não colorretal e não neuroendócrina: Indicações e Resultados em dez pacientes operados. Einstein 2008; 6 (1): 56-62.

22. Chedid AD, Villwock MM, Chedid MF, Rohde L. Fatores prognósticos na ressecção de metástases hepáticas de câncer colorretal. Arq Gastroenterol 2003; 40: 159-65.

23. Oliveira RB, Pinhal MAS, Martins LC, Zampieri JC, Yamagushi N, Sorbello AA, Von Rautenfeld M, Waisberg J. Análise dos parâmetros de sobrevivência em pacientes submetidos à extirpação das metástases hepáticas do carcinoma colo-retal. Rev bras Coloproct 2004; 24 (4): 300-7.

24. Fong Y, Fortner J, Sun RL, Brennan MF, Blumgart LH. Ann Surg 1999; 230 (3): 309-21.

25. Fortner JG, Silva JS, Golbey RB, Cox EB, Maclean BJ. Multivariate analysis of personal series of 247 consecutive patients with liver metastases form colorectal cancer. Treatment by hepatic resection. Ann Surg 1984; 199: 306-16.

26. Scheele J, Stang R, Altendorf-Hofmann A, Paul M. Resection of colorectal liver metastases. World J Surg 1995; 19:59 -71.

27. Gayowski TJ, Iwatsuki S, Madariaga JR, et al. Experience in hepatic resection for metastatic colorectal cancer: analysis of clinical and pathological risk factors. Surgery 1994; 116:703711.

28. Rosen CB, Nagorney DM, Taswell HF, et al. Perioperative blood transfusion and determinants of survival after liver resection for metastatic colorectal carcinoma. Ann Surg 1992; 216:492-505.

29. Nordlinger B, Parc R, Delva E, Quilichini M, Hannoun L, Huguet C. Hepatic resection for colorectal liver metastases. Ann Surg 1987; 205:256-263.

30. Hughes KS, Simons R, Songhorabodi S, et al. Resection of the liver for colorectal carcinoma metastases: a multi-institutional study of indications for resection. Surgery 1988; 103:278 288.

31. Fong Y, Cohen AM, Fortner JG, et al. Liver resection for colorectal metastases. J Clin Oncol 1997; 15:938 -946.
32. Reddy SK, Barbas AS, Marroquin CE, Morse MA, Kuo PC, Clary BM. Resection of noncolorectal nonneuronedocrine liver metastases: a comparative analysis. J Am Coll Surg 2006; 204(3): 372-82.

33. Jaeck D. The significance of hepatic pedicle lymph nodes metastases in surgical management of colorectal liver metastases and of other liver malignancies. Ann Surg Oncol 2003; 10(9): 1007-11.

34. Elias DM, Ouellet JF. Incidence, distribution, and significance of hilar lymph node metastases in hepatic colorectal metastases. Surg Oncol Clin N Am 2003; 12(1): 221-9.

35. Rodgers MS, McCall JL.Surgery for colorectal liver metastases with hepatic lymph node involvement: a systematic review. Br J Surg 2000; 87(9): 1142-55.

36. Sakamoto Y, Yamamoto J, Yoshimoto M, Kasumi F, Kosuge T, Kokudo N, Makuuchi M. Hepatic resection for metastatic breast cancer: prognostic analysis of 34 patients. World J Surg 2005; 29(4):5 24-7.

37. Ercolani G, Grazi GL, Ravaioli M, Ramacciato G, Cescon M, Varotti G, Del Gaudio M, Vetrone G, Pinna AD. The role of liver resections for noncolorectal, nonneuroendocrine metastases: experience with 142 observed cases. Ann Surg Oncol 2005; 12(6): 459-66.

38. Adam R, Chiche L, Aloia T, Elias D, Salmon R, Rivoire M, Jaeck D, Saric J, Le Treut YP, Belghiti J, Mantion G, Mentha G. Hepatic resection for noncolorectal nonendocrine liver metastases: analysis of 1,452 patients and development of a prognostic model. Ann Surg. 2006; 244(4):524-35.

39. Lendoire J, Moro M, Andriani O, Grondona J, Gil O, Raffin $\mathrm{G}$ et al. Liver resection for non-colorectal, non-neuroendocrine metastases: analysis of a multicenter study from Argentina. HPB (Oxford) 2007; 9(6): 435-9.

\section{Endereço para correspondência:}

\section{SERGIO RENATO PAIS COSTA}

Avenida Pacaembu, n. 1400

CEP: $14400-200$

São Paulo - SP - Brasil.

E-mail srenatopaiscosta@hotmail.com 